\title{
Dynamic changes in coagulation parameters and correlation with disease severity and mortality in patients with COVID-19
}

\author{
Wei $\mathrm{Xu}^{1,{ }^{*}}$, Ling Fei ${ }^{1,}$, Chen Lu Huang ${ }^{1,}$, Wei Xia $\mathrm{Li}^{2}, \mathrm{Xu}$ Dong Xie${ }^{1}$, Qiang $\mathrm{Li}^{1}$, Liang Chen ${ }^{1}$ \\ ${ }^{1}$ Department of Liver Diseases, Shanghai Public Health Clinical Center, Fudan University, Shanghai 201508, China \\ ${ }^{2}$ Department of Infectious Diseases, Shanghai Public Health Clinical Center, Fudan University, Shanghai 201508, \\ China \\ ${ }^{*}$ Equal contribution
}

Correspondence to: Qiang Li, Liang Chen; email: liqiang@shphc.org.cn, chenliang@shphc.org.cn

Keywords: COVID-19, SARS-CoV-2, coagulation, disease severity, mortality

Received: November 28, 2020 Accepted: February 17, $2021 \quad$ Published: May 24, 2021

Copyright: (C) $2021 \mathrm{Xu}$ et al. This is an open access article distributed under the terms of the Creative Commons Attribution License (CC BY 3.0), which permits unrestricted use, distribution, and reproduction in any medium, provided the original author and source are credited.

\section{ABSTRACT}

Objective: This study aimed to describe the dynamic changes of coagulation parameters and evaluate the relationship between longitudinal coagulation parameters abnormalities and prognosis of COVID-19 patients.

Methods: We performed a retrospective study of 1131 COVID-19 patients. Longitudinal coagulation parameters and clinical outcomes were analyzed.

Results: Abnormal coagulation parameters were observed in patients with COVID-19, both at hospital admission (INR 2.3\%, PT 7.9\%, APTT 15.4\%, TT 0.9\%, FDP 2.3\%, D-dimer 19.7\%) and peak hospitalization (INR 4.8\%, PT 13.4\%, APTT 25.6\%, TT 2.7\%, FDP 10.4\%, D-dimer 31.5\%). Compared with non-severe patients with COVID-19, severe patients had a slightly higher INR, PT, APTT, whereas remarkably higher FDP and D-dimer $(p<$ 0.05). On multivariate analysis, age > 60 years, male, obesity, comorbidity, abnormal D-dimer on hospital admission, and abnormal peak hospitalization PT, APTT, FDP and D-dimer were associated with COVID-19 severity. The extreme coagulation parameters abnormalities (PT > 16s, FDP > $50 \mathrm{ug} / \mathrm{ml}$, and D-dimer $>5 \mathrm{ug} / \mathrm{ml}$ ) were associated with a significantly higher mortality.

Conclusion: Longitudinal coagulation parameters abnormalities are common in patients with COVID-19, and associated with disease severity and mortality. Monitoring coagulation parameters is advisable to improve the management of patients with COVID-19.

\section{INTRODUCTION}

Coronavirus disease 2019 (COVID-19), which is caused by the severe acute respiratory syndrome coronavirus 2 (SARS-CoV-2) [1], is a new type of infectious respiratory disease, and has become an ongoing global pandemic with a profound impact on society and the global economy. Since November 2019, the outbreak of COVID-19 has influenced more than 200 countries, areas or territories worldwide. As of 22 November 2020, there have been over 57.8 million cases and 1.3 million deaths reported globally since the start of the pandemic [2].
Although COVID-19 is well known for causing substantial respiratory pathology, it can also result in several extrapulmonary manifestations [3]. Nearly $20 \%$ of COVID-19 patients present coagulation abnormalities, which occur more commonly in the severe and critically ill cases [4]. Coagulopathy has been reported in up to $50 \%$ of severe COVID-19 patients, in whom disseminated intravascular coagulation (DIC) has been reported in more than $70 \%$ of the patients [5]. Moreover, coagulopathy appears to be associated with the clinical outcomes of COVID-19 patients. A metaanalysis showed that early coagulation tests can predict higher risk stratification and poorer prognosis in 
patients with COVID-19 [6]. Tang et al. reported that D-dimer and prothrombin time were positively correlated with 28-day mortality of patients with COVID-19 [7]. Yao et al. also reported that D-dimer levels correlate with disease severity and are a reliable prognostic marker for in-hospital mortality in patients admitted for COVID-19 [8].

However, most previous studies described the coagulopathy at hospital admission, the dynamic changes of coagulation parameters during the hospitalization are rare. In addition, few studies have focused on the correlation between abnormal peak hospitalization coagulation parameters and the clinical outcomes of patients with COVID-19. In this study, we aimed to describe the full range of coagulation parameters alterations during the hospital stays and evaluate the relationship between longitudinal coagulation parameters abnormalities and the clinical outcomes of hospitalized patients with COVID-19.

\section{METHODS}

\section{Participants}

A total of 1,131 patients with COVID-19 admitted to Shanghai Public Health Clinical Center, Shanghai, China, between January $20^{\text {th }}, 2020$ and November $10^{\text {th }}$, 2020, were retrospectively analyzed. Shanghai Public Health Clinical Center is a tertiary teaching hospital, and the only designated hospital for adult patients with COVID-19 in Shanghai, China. Among 1,131 enrolled patients, 13 patients were categorized into severe group, and 1,118 patients were categorized into non-severe group at hospital admission. Among 1,118 non-severe patients at hospital admission, 23 patients progressed to severe COVID-19 during the hospital stays, and therefore were switched to severe group. By the end of follow-up (November 10 ${ }^{\text {th }}, 2020$ ), a total of 36 severe patients and 1095 non-severe patients were identified in this study. Among 36 severe patients with COVID-19, 7 died, and 29 were discharged with recovery.

\section{Diagnostic criteria}

Patients with COVID-19 were confirmed based on the detection of SARS-CoV-2 RNA in nasopharyngeal or throat swab specimens using the polymerase chain reaction (PCR) method [9]. Severe patients were defined as any one of the following [9]: (1) Respiratory rates $\geq 30 / \mathrm{min}$; (2) Resting oxygen saturation $\leq 93 \%$; (3) Oxygenation index $\leq 300 \mathrm{mmHg}$; (4) Require mechanical ventilation; (5) shock; (6) Combined with other organ failures and needed the intensive care unit (ICU) admission. Bleeding events were graded according to the modified World Health Organization (WHO) grading system, and included gastrointestinal bleeding, hemoptysis, oral mucosa bleeding, epistaxis, internal bleeding, pulmonary hemorrhage, bleeding from multiple cannulation sites, and intracranial hemorrhage [10].

\section{Data collection}

All data were extracted from the electronic records of Shanghai Public Health Clinical Center. Sociodemographic data were obtained, including age, sex, body mass index (BMI), and underlying diseases. The laboratory tests, chest CT scans, medical management information, clinical outcomes, and length of hospitalization were collected. The following coagulation parameters were recorded at hospital admission and during the hospital stays: prothrombin time (PT), international normalized ratio (INR), activated partial thromboplastin time (APTT), thrombin time (TT), fibrinogen degradation products (FDP), and D-dimer. The coagulation parameters measurements were performed on Stago (STA)RMAX coagulation analyzers and using the original reagents (Startec Diagnostics Co., LTD, France). Coagulation parameters abnormalities were defined as per Shanghai Public Health Clinical Center laboratory reference range standards: INR $>1.2$, PT $>14 \mathrm{~s}$, APTT $>43 \mathrm{~s}$, TT $>21 \mathrm{~s}$, FDP $>5 \mathrm{ug} / \mathrm{ml}$, and D-dimer $>0.5 \mathrm{ug} / \mathrm{ml}$. In this study, the levels of IL- 6 in most of patients were below the low limitation of the human cytokine kit II (Raisecare Ltd, Qingdao, China), and were showed as "0" values in the reports of Shanghai Public Health Clinical Center laboratory. Therefore, in this study, the " 0 " values mean the levels of IL-6 were below the low limitation of detection, rather than the actual test values.

\section{Statistical analysis}

Normal distribution variables were presented using mean and standard deviations, non-normal distribution continuous variables were presented using median and interquartile ranges (IQRs), and qualitative data were presented using frequency distribution. For the comparison of quantitative data between two groups, we used the Student's $t$-test for normal distribution variables, and the non-parametric Mann-Whitney-test for non-normal distribution variables. For the comparison of qualitative variables, we used the chisquared test. Clinical outcomes were modeled using the coagulation parameters at hospital admission and their peak during hospitalization. The multivariate logistic regression analysis was performed to adjust for age, gender, obesity, comorbidity, and the coagulation factors. We performed the survival estimates using the Kaplan-Meier method, comparing the survival curves 
according to the coagulation parameters between the groups.

\section{Ethics approval and consent to participate}

The study was approved by the Clinical Research Ethics Committee of the Shanghai Public Health Clinical Center. The study was conducted in accordance with the principles of the Helsinki declaration of 1975, as revised in 1983.

\section{RESULTS}

\section{Demographic and clinical characteristics of the study population}

The demographic and clinical characteristics of the study population are shown in Table 1 . The median age was 36 years (IQR, 26-50), 690 patients $(61 \%)$ were men, 320 patients $(28.3 \%)$ had obesity, and 202 patients (17.9\%) had comorbidity. The median levels of white blood count (WBC), lymphocyte, platelet, Creactive protein (CRP), erythrocyte sedimentation rate (ESR), interleukin-6 (IL-6), and interleukin-8 (IL-8) were $5.7 \times 10^{9} / \mathrm{L}(\mathrm{IQR}, 4.5-7.1), 1.5 \times 10^{9} / \mathrm{L}(\mathrm{IQR}$, $1.1-2.0), 220 \times 10^{9} / \mathrm{L}(\mathrm{IQR}, 175-265), 0.5 \mathrm{mg} / \mathrm{L}$ (IQR, 0.5-3.8), $26 \mathrm{~mm} / \mathrm{h}$ (IQR, 10-47), $0 \mathrm{pg} / \mathrm{ml}$ (IQR, 0-0), and $1.0 \mathrm{pg} / \mathrm{ml}$ (IQR, 0.3-2.5), respectively. Compared with non-severe patients, severe patients had higher age (median, 64 vs 35 years, $p<0.001$ ), more common male $(77.8 \%$ vs $60.5 \%, p=0.010)$, obesity $(50 \%$ vs $27.6 \%, p=0.001)$, and comorbidity $(69.4 \%$ vs $16.2 \%$, $p<0.001)$. Patients in severe group had a lower lymphocyte $\left(0.8\right.$ vs $\left.1.6 \times 10^{9} / \mathrm{L}, p<0.001\right)$ and platelet (165 vs $\left.222 \times 10^{9} / \mathrm{L}, p<0.001\right)$, but notably higher CRP (36.4 vs $0.5 \mathrm{mg} / \mathrm{L}, p<0.001)$, ESR (49 vs 25 $\mathrm{mm} / \mathrm{h}, p<0.001), \mathrm{IL}-6$ (40.5 vs $0 \mathrm{pg} / \mathrm{ml}, p<0.001)$, and IL-8 levels (8.4 vs $0.8 \mathrm{pg} / \mathrm{ml}, p<0.001)$ than patients in non-severe group (Table 1).

\section{Prophylactic anticoagulation, thrombotic complications, and bleeding events}

During the hospital stays, 55 patients $(4.9 \%)$ received prophylactic anticoagulation, and 17 patients $(1.5 \%)$ received plasma transfusion. Prophylactic anticoagulation therapies were more frequently used in severe patients compared with non-severe patients (97.2\% vs $1.8 \%, p<0.001)$. Patients on prophylactic anticoagulation had higher age, more common comorbidity, higher D-dimer and FDP levels compared with those without anticoagulation (all $p<0.05$ ). Despite prophylactic anticoagulation, we found a radiographically confirmed venous thromboembolism (VTE) rate of $13.8 \%$, pulmonary embolisms (PE) rate of $11.1 \%$, in severe patients with COVID-19 (Table 1).
During the hospital stays, 3 patients $(8.3 \%)$ in severe group and 8 patients $(0.7 \%)$ in non-severe group developed bleeding events (Table 1).

\section{Usage and dosage of prophylactic anticoagulation}

The usage and dosage of prophylactic anticoagulation are shown as following: (1) low molecular weight heparin (LMWH), 5,000 $\mathrm{U}$ daily, subcutaneous injection, 39 patients; (2) enoxaparin, 4,000 IU daily, subcutaneous injection, 10 patients; (3) warfarin, $2.5 \mathrm{mg}$ daily, oral administration, 6 patients. Once patients were confirmed with PE/ VTE, prophylactic anticoagulation therapies were replaced with therapeutic anticoagulation (LMWH, 5,000 U twice daily, or enoxaparin 4,000 IU twice daily).

\section{Coagulation parameters between severe and non- severe patients on hospital admission}

The differences in coagulation parameters between severe and non-severe patients on hospital admission are shown in Table 2. On hospital admission, the severe patients had a slightly higher INR (1.02 vs 0.99, $p=0.016)$, PT (13.6s vs 13.2s, $p=0.019)$, APTT (42.1s vs 38.4s, $p=0.019$ ), whereas remarkably higher FDP (2.03 vs $0.65 \mathrm{ug} / \mathrm{ml}, p<0.001)$ and D-dimer $(0.87$ vs $0.27 \mathrm{ug} / \mathrm{ml}, p<0.001)$ than non-severe patients. Abnormal INR (11.1\% vs $2.0 \%, p<0.001)$, PT $(30.6 \%$ vs $7.1 \%, p<0.001)$, APTT $(47.2 \%$ vs $14.3 \%, p<0.001)$, TT (5.6\% vs $0.7 \%, p=0.002)$, FDP $(13.9 \%$ vs $1.9 \%, p<0.001)$, and D-dimer $(69.4 \%$ vs $18.1 \%, p<0.001)$ were commonly observed in severe patients, compared with non-severe patients. A scatter plots of the coagulation values between severe and non-severe patients on hospital admission are shown in Figure 1.

\section{Hospital admission vs peak hospitalization coagulation parameters}

The prevalence and severity of abnormal coagulation parameters during the hospitalization are shown in Table 3. Abnormal coagulation parameters were observed in patients with COVID-19, both at hospital admission (INR 2.3\%, PT 7.9\%, APTT $15.4 \%$, TT $0.9 \%$, FDP $2.3 \%$, DD 19.7\%) and peak hospitalization (INR 4.8\%, PT 13.4\%, APTT 25.6\%, TT 2.7\%, FDP $10.4 \%$, DD $31.5 \%$ ). Most patients had mild coagulation laboratory parameters changes at hospital admission (INR 1-2 ULN, 92.4\%; PT prolonged 1-3s, 89.9\%; APTT prolonged 1-3s, 63.8\%; TT prolonged 1-3s, 70\%; FDP 1-5 ULN, 73.1\%; D-dimer 1-5 ULN, 91.9\%), as well as peak hospitalization (INR 1-2 ULN, 79.6\%; PT prolonged 1-3s, 87.4\%; APTT prolonged 1$3 \mathrm{~s}, 57.6 \%$; TT prolonged 1-3s, 70\%; FDP 1-5 ULN, 
Table 1. Demographic and clinical characteristics of the study population.

\begin{tabular}{|c|c|c|c|c|}
\hline & Total $(n=1131)$ & Non-severe $(n=1095)$ & Severe $(n=36)$ & $p$-value \\
\hline Age (years) & $36(26-50)$ & $35(26-49)$ & $64(50-73)$ & $<0.001$ \\
\hline Male, $n(\%)$ & $690(61 \%)$ & $662(60.5 \%)$ & $28(77.8 \%)$ & 0.010 \\
\hline Obesity, $n(\%)$ & $320(28.3 \%)$ & $302(27.6 \%)$ & $18(50 \%)$ & 0.001 \\
\hline Comorbidity, $n(\%)$ & $202(17.9 \%)$ & $177(16.2 \%)$ & $25(69.4 \%)$ & $<0.001$ \\
\hline Hypertension & $126(11.1 \%)$ & $107(9.8 \%)$ & $19(52.8 \%)$ & $<0.001$ \\
\hline Diabetes & $58(5.1 \%)$ & $50(4.6 \%)$ & $8(22.2 \%)$ & $<0.001$ \\
\hline \multicolumn{5}{|l|}{ Laboratory findings } \\
\hline WBC $\left(10^{9} / \mathrm{L}\right)$ & $5.7(4.5-7.1)$ & $5.7(4.5-7.1)$ & $5.9(3.8-7.3)$ & 0.503 \\
\hline $\operatorname{LYMP}\left(10^{9} / \mathrm{L}\right)$ & $1.5(1.1-2.0)$ & $1.6(1.2-2.0)$ & $0.8(0.5-1.1)$ & $<0.001$ \\
\hline Platelet $\left(10^{9} / \mathrm{L}\right)$ & $220(175-265)$ & $222(177-266)$ & $165(123-206)$ & $<0.001$ \\
\hline CRP (mg/L) & $0.5(0.5-3.8)$ & $0.5(0.5-2.9)$ & $36.4(9.5-81.9)$ & $<0.001$ \\
\hline $\operatorname{ESR}(\mathrm{mm} / \mathrm{h})$ & $26(10-47)$ & $25(10-46)$ & $49(37-89)$ & $<0.001$ \\
\hline IL-6 (pg/ml) & $0(0-0)$ & $0(0-0)$ & $40.5(24.6-80.4)$ & $<0.001$ \\
\hline IL-8 (pg/ml) & $1.0(0.3-2.5)$ & $0.8(0.2-2.3)$ & $8.4(3.2-25.6)$ & $<0.001$ \\
\hline \multicolumn{5}{|c|}{ Prophylactic Anticoagulation and Thrombotic Complications } \\
\hline Anticoagulation & $55(4.9 \%)$ & $20(1.8 \%)$ & $35(97.2 \%)$ & $<0.001$ \\
\hline Plasma transfusion & $17(1.5 \%)$ & $2(0.2 \%)$ & $15(41.7 \%)$ & $<0.001$ \\
\hline VTE & $9(0.8 \%)$ & $2(0.2 \%)$ & $7(19.4 \%)$ & $<0.001$ \\
\hline PE & $4(0.35 \%)$ & 0 & $4(11.1 \%)$ & $<0.001$ \\
\hline Bleeding events & $2(0.2 \%)$ & 0 & $2(5.6 \%)$ & $<0.001$ \\
\hline
\end{tabular}

Abbreviations: WBC: white blood count; LYMP: lymphocyte; CRP: C-reactive protein; ESR: erythrocyte sedimentation rate; VTE: venous thromboembolism; PE: Pulmonary embolism. The $p$ values indicate differences between severe group and non-severe group.

74.6\%; D-dimer 1-5 ULN, 80.1\%). The severe coagulation laboratory changes were relatively infrequently observed at hospital admission (INR > 3 ULN, 3.8\%; PT prolonged $>6 \mathrm{~s}, 4.5 \%$; APTT prolonged $>6 \mathrm{~s}, 22.4 \%$; TT prolonged > 6s, 10\%; FDP > $10 \mathrm{ULN}$, $15.4 \%$; D-dimer > $10 \mathrm{ULN}, 4.5 \%$ ), as well as peak hospitalization (INR > 3 ULN, 14.8\%; PT prolonged > 6s, 6.0\%; APTT prolonged > 6s, 26.2\%; TT prolonged $>$ 6s, 16.7\%; FDP > $10 \mathrm{ULN}, 16.1 \%$; D-dimer > 10 ULN, 12.4\%).

Coagulation parameters classified by aged/non-aged, obese/non-obese, oncologic/non-oncologic, and diabetic/non-diabetic

We analyzed coagulation parameters abnormalities on hospital admission classified by aged/non-aged, obese/non-obese, oncologic/non-oncologic, and diabetic/non-diabetic in Table 4 . The results showed that coagulation parameters abnormalities were commonly observed in patients with age > 60 years, diabetes or malignant tumour compared with patients without the above situations $(p<0.05)$. There is no significant difference between obese patients and non-obese patients in all coagulation parameters abnormalities $(p>0.05)$ (Table 4).

\section{Correlations between hematologic parameters and coagulation parameters}

The correlation between hematologic parameters and coagulation parameters was analyzed using the Spearman test in Table 5. The results showed that lymphocyte is negatively correlated with APTT ( $\mathrm{r}=-$ $0.13, p<0.001)$, FDP $(\mathrm{r}=-0.10, p=0.001)$, and Ddimer ( $\mathrm{r}=-0.14, p<0.001$ ); $\mathrm{CRP}$ is positively correlated with APTT $(\mathrm{r}=0.20, p<0.001)$, FDP $(\mathrm{r}=$ $0.30, p<0.001)$, and D-dimer $(\mathrm{r}=0.31, p<0.001)$; ESR is positively correlated with FDP $(\mathrm{r}=0.11, p<$ $0.001)$ and $\mathrm{D}$-dimer $(\mathrm{r}=0.17, p<0.001)$; IL-6 is positively correlated with PT $(\mathrm{r}=0.25, p<0.001)$, APTT $(\mathrm{r}=0.28, p<0.001)$, FDP $(\mathrm{r}=0.42, p<0.001)$, and D-dimer ( $\mathrm{r}=0.45, p<0.001)$; IL-8 is positively correlated with PT $(\mathrm{r}=0.23, p<0.001)$, APTT $(\mathrm{r}=$ $0.24, p<0.001)$, FDP $(\mathrm{r}=0.38, p<0.001)$, and Ddimer $(r=0.40, p<0.001)$. 
Table 2. Coagulation parameters between severe and non-severe patients on hospital admission.

\begin{tabular}{lcccc}
\hline & Total & Non-severe & Severe & $p$-value \\
\hline Number & 1131 & 1095 & 36 & \\
INR & $0.99(0.95-1.04)$ & $0.99(0.95-1.04)$ & $1.02(0.96-1.08)$ & 0.016 \\
\multicolumn{1}{c}{ Abnormal (> 1.2) } & $26(2.3 \%)$ & $22(2.0 \%)$ & $4(11.1 \%)$ & $<0.001$ \\
PT (s) & $13.0(13.0-14.0)$ & $13.2(12.8-13.7)$ & $13.6(12.9-14.1)$ & 0.019 \\
$\quad$ Abnormal (> 14s) & $89(7.9 \%)$ & $78(7.1 \%)$ & $11(30.6 \%)$ & $<0.001$ \\
APTT (s) & $38.5(35.7-41.5)$ & $38.4(35.7-41.5)$ & $42.1(35.7-49.4)$ & 0.001 \\
$\quad$ Abnormal (> 43s) & $174(15.4 \%)$ & $157(14.3 \%)$ & $17(47.2 \%)$ & $<0.001$ \\
TT (s) & $16.3(15.7-17.1)$ & $16.3(15.7-17.1)$ & $16.4(15.8-18.1)$ & 0.194 \\
$\quad$ Abnormal (> 21s) & $10(0.9 \%)$ & $8(0.7 \%)$ & $2(5.6 \%)$ & 0.002 \\
FDP (ug/ml) & $0.70(0.20-1.40)$ & $0.65(0.22-1.37)$ & $2.03(1.13-3.68)$ & $<0.001$ \\
$\quad$ Abnormal (> 5 ug/ml) & $26(2.3 \%)$ & $21(1.9 \%)$ & $5(13.9 \%)$ & $<0.001$ \\
D-dimer (ug/ml) & $0.28(0.20-0.44)$ & $0.27(0.20-0.42)$ & $0.87(0.47-1.41)$ & $<0.001$ \\
$\quad$ Abnormal (> 0.5 ug/ml) & $223(19.7 \%)$ & $198(18.1 \%)$ & $25(69.4 \%)$ & $<0.001$ \\
\hline
\end{tabular}

Abbreviations: INR: international normalized ratio; PT: prothrombin time; APTT: activated partial thromboplastin time; TT: thrombin time; FDP: fibrinogen degradation products. The $p$ values indicate differences between severe group and non-severe group.

\section{Association between coagulation parameters and clinical outcomes}

The association between admission and peak hospitalization coagulation parameters and clinical outcomes is summarized in Table 6. On multivariate analysis, age > 60 years, male, obesity, comorbidity, abnormal D-dimer on hospital admission, and abnormal peak hospitalization PT, APTT, FDP, and D-dimer were associated with severe COVID-19 (OR $>1 ; p<0.05$ ).
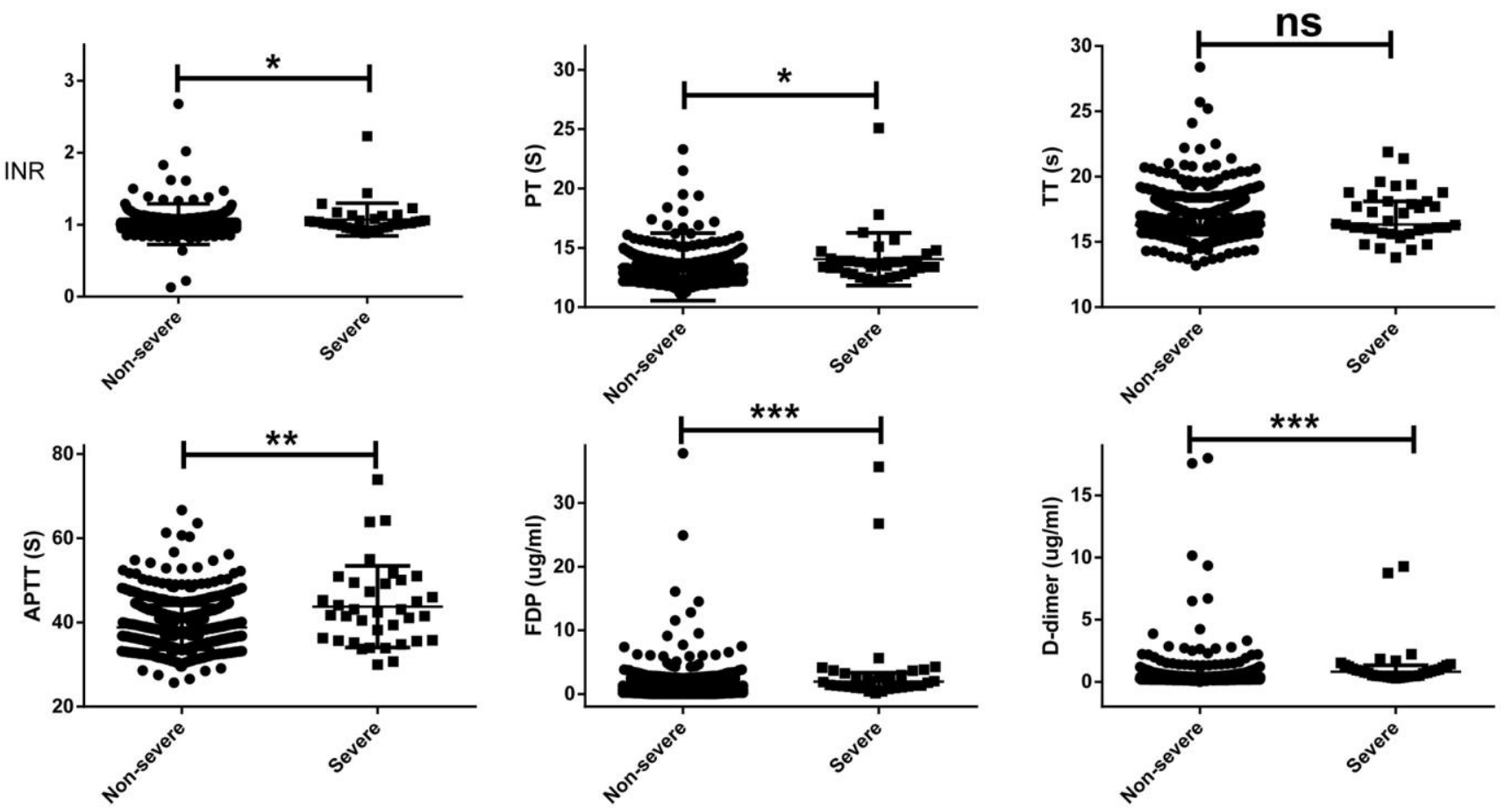

Figure 1. A scatter plots of the coagulation values on hospital admission between severe and non-severe patients. The severe patients had a slightly higher INR (1.02 vs 0.99, $p=0.016)$, PT (13.6s vs 13.2s, $p=0.019)$, APTT ( $42.1 \mathrm{~s}$ vs $38.4 \mathrm{~s}, p=0.019)$, whereas remarkably higher FDP (2.03 vs $0.65 \mathrm{ug} / \mathrm{ml}, p<0.001)$ and $D$-dimer $(0.87$ vs $0.27 \mathrm{ug} / \mathrm{ml}, p<0.001)$ than non-severe patients. 
Table 3. Hospital admission vs peak hospitalization coagulation parameters in 1131 patients.

\begin{tabular}{|c|c|c|c|}
\hline & & Hospital admission & Peak Hospitalization \\
\hline \multirow[t]{4}{*}{ INR } & Abnormal (> 1.2) & $26(2.3 \%)$ & $54(4.8 \%)$ \\
\hline & $1-2$ ULN & $24(92.4 \%)$ & $43(79.6 \%)$ \\
\hline & $2-3 \mathrm{ULN}$ & $1(3.8 \%)$ & $3(5.6 \%)$ \\
\hline & $>3 \mathrm{ULN}$ & $1(3.8 \%)$ & $8(14.8 \%)$ \\
\hline \multirow[t]{4}{*}{ PT (s) } & Abnormal (> 14s) & $89(7.9 \%)$ & $151(13.4 \%)$ \\
\hline & Prolonged 1-3s & $80(89.9 \%)$ & $132(87.4 \%)$ \\
\hline & Prolonged 4-6s & $5(5.6 \%)$ & $10(6.6 \%)$ \\
\hline & Prolonged $>6 s$ & $4(4.5 \%)$ & $9(6.0 \%)$ \\
\hline \multirow[t]{4}{*}{ APTT (s) } & Abnormal (> 43s) & $174(15.4 \%)$ & $290(25.6 \%)$ \\
\hline & Prolonged 1-3s & $111(63.8 \%)$ & $167(57.6 \%)$ \\
\hline & Prolonged 4-6s & $24(13.8 \%)$ & $47(16.2 \%)$ \\
\hline & Prolonged $>6 \mathrm{~s}$ & $39(22.4 \%)$ & $76(26.2 \%)$ \\
\hline \multirow[t]{4}{*}{ TT (s) } & Abnormal (> 21s) & $10(0.9 \%)$ & $30(2.7 \%)$ \\
\hline & Prolonged 1-3s & $7(70 \%)$ & $21(70 \%)$ \\
\hline & Prolonged 4-6s & $2(20 \%)$ & $4(13.3 \%)$ \\
\hline & Prolonged $>6 \mathrm{~s}$ & $1(10 \%)$ & $5(16.7 \%)$ \\
\hline \multirow[t]{4}{*}{ FDP (ug/ml) } & Abnormal (> 5 ug/ml) & $26(2.3 \%)$ & $118(10.4 \%)$ \\
\hline & $1-5 \mathrm{ULN}$ & $19(73.1 \%)$ & $88(74.6 \%)$ \\
\hline & 5-10 ULN & $3(11.5 \%)$ & $11(9.3 \%)$ \\
\hline & $>10 \mathrm{ULN}$ & $4(15.4 \%)$ & $19(16.1 \%)$ \\
\hline \multirow[t]{4}{*}{ D-dimer (ug/ml) } & Abnormal (> $0.5 \mathrm{ug} / \mathrm{ml})$ & $223(19.7 \%)$ & $356(31.5 \%)$ \\
\hline & $1-5 \mathrm{ULN}$ & $205(91.9 \%)$ & $285(80.1 \%)$ \\
\hline & 5-10 ULN & $8(3.6 \%)$ & $27(7.6 \%)$ \\
\hline & $>10 \mathrm{ULN}$ & $10(4.5 \%)$ & $44(12.4 \%)$ \\
\hline
\end{tabular}

Abbreviations: INR: international normalized ratio; PT: prothrombin time; APTT: activated partial thromboplastin time; TT: thrombin time; FDP: fibrinogen degradation products; ULN: upper limit of normal. The ULNs of coagulation parameters were defined as per Shanghai Public Health Clinical Center laboratory reference range standards: INR $\leq 1.2$, PT $\leq 14 \mathrm{~s}$, APTT $\leq 43 \mathrm{~s}$, $T \mathrm{~T} \leq 21 \mathrm{~s}, \mathrm{FDP} \leq 5 \mathrm{ug} / \mathrm{ml}$, D-dimer $\leq 0.5 \mathrm{ug} / \mathrm{ml}$.

The dynamic profile of coagulation parameters in patients by severity of COVID-19 is illustrated in Figure 2. Compared with non-severe patients, severe COVID-19 patients had markedly higher levels of INR, PT, APTT, FDP, and D-dimer from baseline to 30 days after admission $(p<0.05)$. On multivariate analysis, age $>60$ years, obesity, comorbidity, and abnormal peak hospitalization PT $(\mathrm{OR}=3.32 ; 95 \%$ CI 1.43-24.94; $p=$ $0.001)$, FDP (OR $=2.63 ; 95 \%$ CI $1.16-5.57 ; p=0.032)$, and $\mathrm{D}$-dimer $(\mathrm{OR}=3.21 ; 95 \% \mathrm{CI} 1.32-21.65 ; p=$ 0.026 ) were associated with death. Kaplan-Meier curves for cumulative rate of survival during hospitalization in patients with different level of PT (a), FDP (b), and Ddimer (c) are illustrated in Figure 3.

\section{Further analyzed patients had significant increase in PT and D-dimer}

In this study, 151 patients had increased PT during the hospital stays, among them, 2 patients developed bleeding events. The 2 patients with bleeding events were severe cases and on anticoagulation. The bleeding rates of patients receiving anticoagulants is $2 / 55$ (3.6\%). In our cohort, 44 patients had the significant increase in 
Table 4. Coagulation parameters on hospital admission classified by aged/non-aged, obese/non-obese, oncologic/non-oncologic, and diabetic/non-diabetic.

\begin{tabular}{|c|c|c|c|c|c|c|c|c|c|c|c|c|}
\hline & \multicolumn{3}{|c|}{ Age (years) } & \multicolumn{3}{|c|}{ Obesity } & \multicolumn{3}{|c|}{ Malignant tumour } & \multicolumn{3}{|c|}{ Diabetes } \\
\hline & $\leq 60$ & $>60$ & & With & Without & & With & Without & & With & Without & \\
\hline & $(n=1003)$ & $(n=128)$ & $P$ & $(n=320)$ & $(n=811)$ & $p$ & $(n=8)$ & $(n=1123)$ & $p$ & $(n=58)$ & $(n=1073)$ & $p$ \\
\hline $\begin{array}{c}\text { Abnormal } \\
\text { INR }\end{array}$ & $19(1.9 \%)$ & $7(5.5 \%)$ & 0.011 & $5(1.6 \%)$ & $21(2.6 \%)$ & 0.299 & $2(25 \%)$ & $24(2.1 \%)$ & $<0.001$ & $5(8.6 \%)$ & $21(2.0 \%)$ & 0.001 \\
\hline $\begin{array}{c}\text { Abnormal } \\
\text { PT }\end{array}$ & $\begin{array}{c}729 \\
(7.2 \%)\end{array}$ & $17(13.3 \%)$ & 0.016 & $\begin{array}{c}15 \\
(4.7 \%)\end{array}$ & $\begin{array}{c}74 \\
(9.1 \%)\end{array}$ & 0.130 & $\begin{array}{c}2 \\
(25 \%)\end{array}$ & $\begin{array}{c}87 \\
(7.8 \%)\end{array}$ & $<0.001$ & $\begin{array}{c}10 \\
(17.2 \%)\end{array}$ & $\begin{array}{c}79 \\
(7.4 \%)\end{array}$ & 0.007 \\
\hline $\begin{array}{c}\text { Abnormal } \\
\text { APTT }\end{array}$ & $\begin{array}{c}145 \\
(14.5 \%)\end{array}$ & $29(22.7 \%)$ & 0.016 & $\begin{array}{c}52 \\
(16.3 \%)\end{array}$ & $\begin{array}{c}122 \\
(15.0 \%)\end{array}$ & 0.612 & $2(25 \%)$ & $\begin{array}{c}172 \\
(15.3 \%)\end{array}$ & $<0.001$ & $\begin{array}{c}24 \\
(41.4 \%)\end{array}$ & $\begin{array}{c}150 \\
(14.0 \%)\end{array}$ & $<0.001$ \\
\hline $\begin{array}{c}\text { Abnormal } \\
\text { TT }\end{array}$ & $5(0.5 \%)$ & $5(3.9 \%)$ & $<0.001$ & $3(0.9 \%)$ & $5(0.6 \%)$ & 0.562 & $1(12.5 \%)$ & $9(0.8 \%)$ & $<0.001$ & $3(5.2 \%)$ & $7(0.7 \%)$ & $<0.001$ \\
\hline $\begin{array}{c}\text { Abnormal } \\
\text { FDP }\end{array}$ & $17(1.7 \%)$ & $9(7.0 \%)$ & $<0.001$ & $8(2.5 \%)$ & $18(2.2 \%)$ & 0.777 & $2(25 \%)$ & $25(22.3 \%)$ & $<0.001$ & $4(6.9 \%)$ & $22(2.1 \%)$ & 0.017 \\
\hline $\begin{array}{c}\text { Abnormal } \\
\text { D-dimer }\end{array}$ & $\begin{array}{c}161 \\
(16.1 \%)\end{array}$ & $62(48.4 \%)$ & $<0.001$ & $\begin{array}{c}72 \\
(22.5 \%)\end{array}$ & $\begin{array}{c}151 \\
(18.6 \%)\end{array}$ & 0.140 & $\begin{array}{c}4 \\
(50 \%)\end{array}$ & $\begin{array}{c}219 \\
(19.5 \%)\end{array}$ & 0.031 & $\begin{array}{c}25 \\
(43.1 \%)\end{array}$ & $\begin{array}{c}198 \\
(18.5 \%)\end{array}$ & $<0.001$ \\
\hline
\end{tabular}

Abbreviations: INR: international normalized ratio; PT: prothrombin time; APTT: activated partial thromboplastin time; TT: thrombin time; FDP: fibrinogen degradation products.

Table 5. Correlations between hematologic parameters and coagulation parameters on admission.

\begin{tabular}{ccccccccccc}
\hline & \multicolumn{2}{c}{ PT } & \multicolumn{2}{c}{ APTT } & \multicolumn{2}{c}{ FDP } & \multicolumn{2}{c}{ D-dimer } & \multicolumn{2}{c}{ TT } \\
\cline { 2 - 11 } & $\mathbf{r}$ & $\boldsymbol{p}$ & $\mathbf{r}$ & $\boldsymbol{p}$ & $\mathbf{r}$ & $\boldsymbol{p}$ & $\mathbf{r}$ & $\boldsymbol{p}$ & $\mathbf{r}$ & $\boldsymbol{p}$ \\
\hline WBC & 0.02 & 0.554 & -0.04 & 0.171 & 0.18 & $<0.001$ & 0.11 & $<0.001$ & 0.02 & 0.474 \\
Platelet & 0.02 & 0.447 & -0.06 & 0.097 & 0.01 & 0.729 & -0.04 & 0.177 & 0.05 & 0.109 \\
LYMP & -0.01 & 0.830 & -0.13 & $<0.001$ & -0.10 & 0.001 & -0.14 & $<0.001$ & 0.01 & 0.763 \\
CRP & 0.02 & 0.492 & 0.20 & $<0.001$ & 0.30 & $<0.001$ & 0.31 & $<0.001$ & -0.01 & 0.690 \\
PCT & 0.02 & 0.578 & 0.01 & 0.652 & -0.01 & 0.873 & -0.01 & 0.869 & 0.01 & 0.639 \\
ESR & -0.20 & 0.605 & 0.04 & 0.148 & 0.11 & $<0.001$ & 0.17 & $<0.001$ & -0.01 & 0.938 \\
IL-6 & 0.25 & $<0.001$ & 0.28 & $<0.001$ & 0.42 & $<0.001$ & 0.45 & $<0.001$ & 0.04 & 0.188 \\
IL-8 & 0.23 & $<0.001$ & 0.24 & $<0.001$ & 0.38 & $<0.001$ & 0.40 & $<0.001$ & 0.03 & 0.216 \\
\hline
\end{tabular}

Abbreviations: WBC: white blood count; LYMP: Lymphocyte; CRP: C-reactive protein; PCT: procalcitonin; ESR: erythrocyte sedimentation rate; PT: prothrombin time; APTT: activated partial thromboplastin time; FDP: fibrinogen degradation products; TT: thrombin time; r: correlation coefficient.

D-dimer (> $10 \mathrm{ULN})$, among them, 17 patients (38.6\%) had the serious illness, and 5 patients (11.4\%) died. Cox regression analysis showed the significant increase in D-dimer ( $>10$ ULN) was associated with serious illness $(\mathrm{HR}=22 ; \mathrm{CI} \%=6-117 ; p<0.001)$, and a higher mortality $(\mathrm{HR}=64 ; \mathrm{CI} \%=13-334 ; p<0.001)$.

\section{DISCUSSION}

This study aimed to describe the dynamic changes of coagulation parameters during hospitalization, and evaluate the association between longitudinal coagulation parameters abnormalities and clinical outcomes of COVID-19 patients. The strength of this study included its large sample size, and the longitudinal coagulation parameters description at admission and during hospitalization. Although the differences in coagulation parameters were not always striking (some moving within the ULN), Figure 2 clearly showed that severe COVID-19 patients had higher levels of coagulation parameters than non-severe patients from baseline to 30 days after admission. Figure 3 clearly showed that peak hospitalization PT, FDP, and D-dimer were associated with mortality of COVID-19 patients.

Previous studies also indicated the association between abnormal coagulation parameters and the poor prognosis of COVID-19 patients [11]. In the study published by Quintana-Diaz et al, non-survivors showed a more than 3.5-fold increase in D-dimer compared with the survivors [12]. Yao et al. reported that median dimer 
Table 6. Association between coagulation parameters and clinical outcomes (Multivariate model).

\begin{tabular}{|c|c|c|c|c|}
\hline & \multicolumn{2}{|c|}{ Severe COVID-19 } & \multicolumn{2}{|l|}{ Death } \\
\hline & OR $(90 \%$ CI $)$ & $p$-value & OR $(90 \%$ CI $)$ & $p$-value \\
\hline Age $>60$ years & $3.48(1.33-9.12)$ & 0.011 & $6.01(1.78-15.27)$ & 0.007 \\
\hline Male & $2.85(1.08-7.53)$ & 0.035 & $1.60(0.31-8.26)$ & 0.577 \\
\hline Obesity & $3.05(1.13-8.21)$ & 0.028 & $1.75(1.21-4.32)$ & 0.028 \\
\hline Comorbidity & $3.93(1.55-9.95)$ & 0.004 & $6.51(2.83-19.32)$ & $<0.001$ \\
\hline \multicolumn{5}{|l|}{ Hospital Admission } \\
\hline Abnormal INR & $0.62(0.09-4.53)$ & 0.638 & $0.42(0.02-7.46)$ & 0.515 \\
\hline Abnormal PT & $0.53(0.11-2.65)$ & 0.438 & $1.17(0.35-3.46)$ & 0.280 \\
\hline Abnormal APTT & $1.36(0.46-4.02)$ & 0.579 & $1.67(0.48-5.18)$ & 0.374 \\
\hline Abnormal TT & $0.45(0.03-7.55)$ & 0.576 & $2.23(0.47-8.25)$ & 0.127 \\
\hline Abnormal FDP & $2.34(0.56-9.83)$ & 0.245 & $0.24(0.01-2.55)$ & 0.424 \\
\hline Abnormal D-dimer & $1.58(1.16-4.18)$ & 0.035 & $0.74(0.07-3.59)$ & 0.612 \\
\hline \multicolumn{5}{|l|}{ Peak Hospitalization } \\
\hline Abnormal INR & $0.89(0.70-5.14)$ & 0.208 & $0.98(0.37-18.19)$ & 0.874 \\
\hline Abnormal PT & $2.07(1.18-5.97)$ & $<0.001$ & $3.32(1.43-24.94)$ & 0.001 \\
\hline Abnormal APTT & $3.51(1.39-8.85)$ & 0.008 & $2.33(0.54-8.46)$ & 0.127 \\
\hline Abnormal TT & $2.53(0.73-8.58)$ & 0.145 & $0.31(0.02-5.71)$ & 0.577 \\
\hline Abnormal FDP & $2.50(1.04-6.27)$ & 0.041 & $2.63(1.16-5.57)$ & 0.032 \\
\hline Abnormal D-dimer & $3.85(1.42-8.46)$ & 0.012 & $3.21(1.32-21.65)$ & 0.026 \\
\hline
\end{tabular}

Abbreviations: OR: odds ratio; $\mathrm{Cl}$ : confidence interval; INR: international normalized ratio; PT: prothrombin time; APTT: activated partial thromboplastin time; TT: thrombin time; FDP: fibrinogen degradation products.

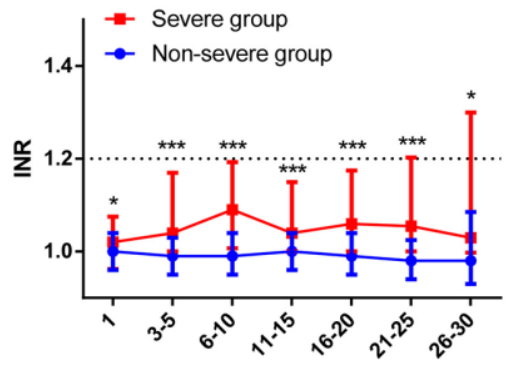

Days After Disease Onset

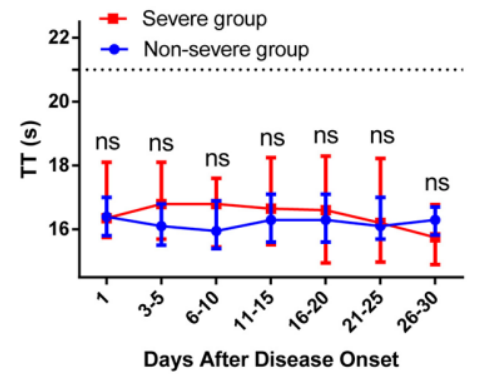

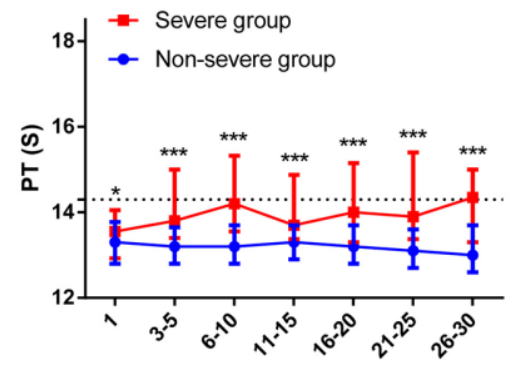

Days After Disease Onset

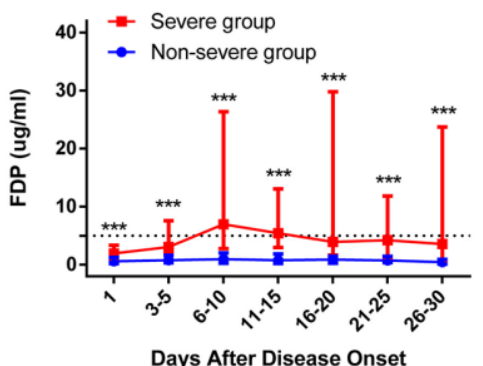

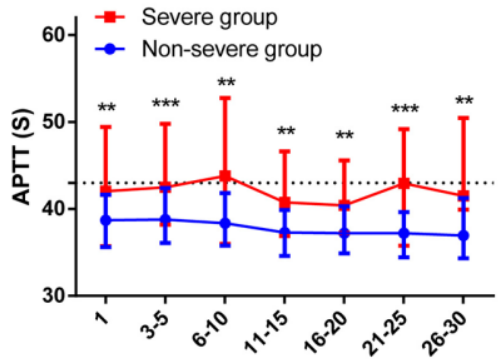

Days After Disease Onset

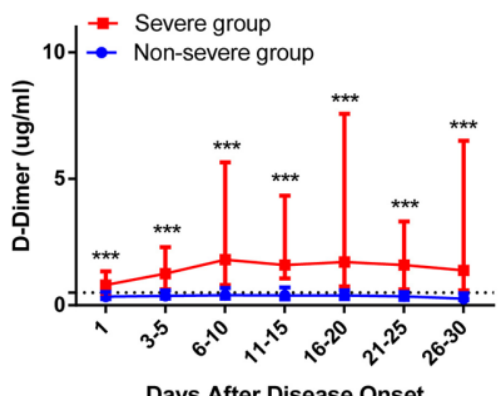

\begin{tabular}{|l|l|l|l|l|l|l|l|}
\hline Time (days) & 1 & $3-5$ & $6-10$ & $11-15$ & $16-20$ & $21-25$ & $26-30$ \\
\hline Severe group $(\mathrm{n})$ & 36 & 35 & 34 & 30 & 29 & 26 & 14 \\
\hline Non- severe group $(\mathrm{n})$ & 1095 & 634 & 590 & 348 & 171 & 80 & 26 \\
\hline
\end{tabular}

Figure 2. Dynamic profile of coagulation parameters in patients by severity of COVID-19. The coagulation parameters in the nonsevere group (blue line) and severe group (red line) were analyzed at different time points after hospital admission. The coagulation parameters are shown using median and IQR. 
D-in non-survivors was significantly higher than that in survivors [6.21 vs $1.02 \mathrm{mg} / \mathrm{L}, p<0.001$ ], and D-dimer of $>2.14 \mathrm{mg} / \mathrm{L}$ predicted in-hospital mortality with a sensitivity of $88.2 \%$ and specificity of $71.3 \%$ [8]. A meta-analysis showed the risk of mortality was fourfold higher in patients with high $\mathrm{D}$-dimer vs. normal Ddimer (risk ratio 4.11, 95\% CI $2.48-6.84, p<0.001$ ) and the risk of developing severe disease was two-fold higher in patients with high D-dimer vs. normal Ddimer (risk ratio $2.04,95 \%$ CI $1.34-3.11, p<0.001$ ) [13].

Though the specific mechanisms are still unclear, SARS-CoV-2 obviously involves potentially deleterious processes in hemostasis/coagulation system. The potential causes of coagulopathy and fibrinolytic

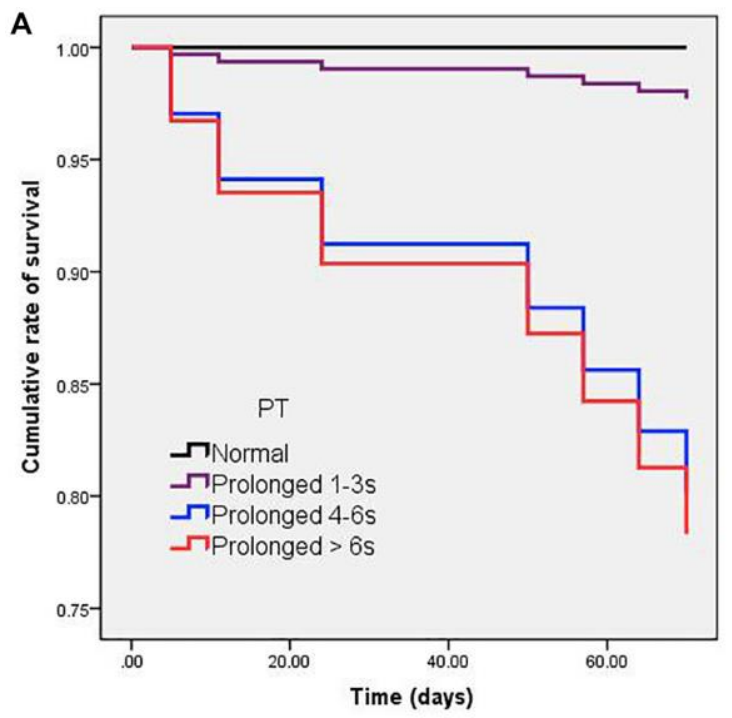

disruption in patients with COVID-19 are shown as following: (1) Dysfunctional angiotensin converting enzyme 2 (ACE2). Dysfunction of ACE2 leads to abnormal renin-angiotensin (RAS) system activation, which promotes platelet adhesion and aggregation and enhances the risk of thromboembolism following the invasion of SARS-CoV-2 [14]. (2) Innate immune response. In fact, the regulation of coagulation and innate immunity is intertwined because they share some common pathways in response to viral invasion, such as the function of tissue factor in the initiation of procoagulation and the host immune response [15]. (3) Inflammatory factor storm. Inflammation due to SARSCoV-2 infection aggravates various proinflammatory cytokines, which increase the expression of tissue factor and von Willebrand factor from endothelial cells and
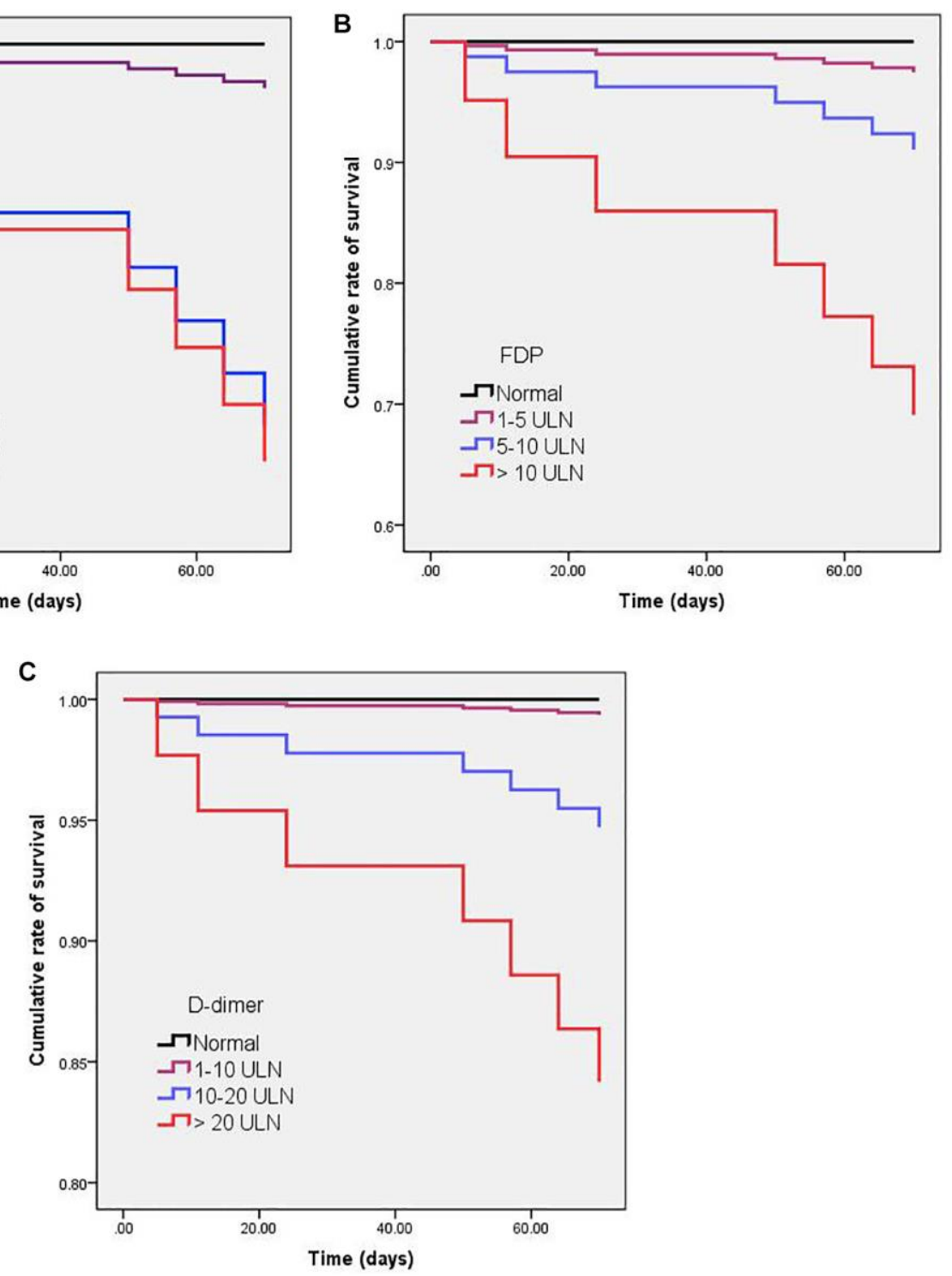

Figure 3. Kaplan-Meier curves for cumulative rate of survival in patients with different levels of PT (A), FDP (B), and D-dimer (C). Cox regression analysis showed that PT prolonged $>4 \mathrm{~s}$ (HR = 90; Cl\% $=20-404 ; p<0.001)$, D-dimer $>10$ ULN (HR $=64 ; \mathrm{Cl} \%=$ $13-334 ; p<0.001)$, and FDP > 10 ULN (HR $=48 ; C l \%=11-214 ; p<0.001)$ were significantly associated with a higher mortality. 
monocytes, promoting platelet aggregation and initiating the clotting cascade [14]. Besides, proinflammatory cytokines can also suppress the synthesis of anticoagulants and fibrinolysis by downregulating thrombomodulin and endothelial protein $\mathrm{C}$ receptor and upregulating plasminogen activator inhibitor-1(PAI-1) levels, which will finally activate coagulation cascade and inhibit fibrinolytic reaction [16]. (4) Endothelial cell infection and endotheliitis. Rapidly emerging data are providing insight into how endothelial dysfunction may contribute to the coagulopathy in patients with COVID-19 [17].

In this study, among 36 severe patients, 35 (97.2\%) received prophylactic anticoagulation. Despite prophylactic anticoagulation, we found a radiographically confirmed VTE rate of $19.4 \%$, PE rate of $11.1 \%$, in severe patients with COVID-19. Helms et al. also reported that despite anticoagulation, a number of patients with ARDS secondary to COVID-19 developed thrombotic complications, mainly PE (16.7\%) [18]. Although this study and other study find clotting events in COVID-19 patients with prophylactic anticoagulation, we declare that anticoagulation helps to reduce the risk of clotting events in severe and critically ill COVID-19 patients. Perhaps the coagulation burden would be even higher if not on the anticoagulation. For example, Cui et al. reported that the overall incidence of VTE was $25 \%$ in severe COVID-19 patients without anticoagulation [19]. Llitjos et al. reported the overall rate of VTE was $69 \%$ in severe COVID-19 patients [20]. According to a consensus statement for prevention and treatment of VTE associated with COVID-19, all severe and critically ill COVID-19 patients have a high risk of VTE, so anticoagulation is strongly recommended in absence of contraindication [4].

In this study, the incidence rate of bleeding events in patients receiving anticoagulants is $2 / 55(3.6 \%)$, which is consistent with previous studies. Two groups with patients receiving anticoagulants reported bleeding rates of $0 \%$ to $3 \%$ [21, 22]. Paranjpe et al. compared systemic anticoagulation $v s$ no anticoagulation in 2772 patients with COVID-19, and found no difference in bleeding events $(1.9 \%$ vs 3\%; $p>0.05)$ [22]. Tafur et al. analyzed 1496 patients received anticoagulation therapy, and found that the 3-month cumulative incidence rates of major and overall bleeding were $2.1 \%$ and $5.1 \%$, respectively [23]. In conclusion, a few reports exist regarding bleeding outcomes for hospitalized patients receiving either prophylaxis or therapeutic anticoagulation, which may reduce venous thrombotic events while slightly increasing bleeding events. Decision making requires a careful balance between these 2 outcomes to maximize net clinical benefit for these patients.
Elevated levels of inflammation-related cytokines, including IL-6, IL-8, and IL-10 were found in patients with COVID-19 [24]. In this study, we found that IL-6 and IL-8 levels are positively correlated with abnormal PT, APTT, FDP, and D-dimer. The results showed the potential of the intimate interconnection with inflammatory disorders, hypercoagulation and excessive immunity following SARS-CoV-2 invasion in dysfunctional coagulation. The stimulation of an immune response and proinflammation activate the coagulation cascade, and blood clotting in turn orchestrates the pathway of an excessive inflammatory response [14].

The limitations of this study are evident. First, retrospective observational cohort study design with inclusion restricted to patients who were hospitalized within a single hospital, and limited access to laboratory, and medication variables which may influence clinical outcomes. Second, although the study included 1131 patients with COVID-19, severe cases and death cases occurred in a small number of patients, resulting in wider confidence intervals for the ORs and HRs describing associations with these outcomes. Third, we could not construct a risk model of thrombosis. Due to prophylactic anticoagulation, only 13 patients developed thrombosis. The number of patients with thrombosis is not enough to explore the risk factors of thrombosis. On addition, because of the rapid control of the outbreak in China, in a short period, we could not get a validation cohort, which is necessary to evaluate the performance of a risk model of thrombosis. Fourth, the criteria of severe was a retrospective one rather than clinical. The CT tests were not done on all patients to rule out PE, and the vascular ultrasound tests were not done on all patients to rule out VTE as well. Therefore, data from PE and VTE is too small to include separately.

In conclusion, longitudinal coagulation parameters abnormalities are common in COVID-19 patients, and associated with disease severity and mortality. Abnormal peak hospitalization PT, FDP, and D-dimer were associated with a higher mortality of patients with COVID-19. Monitoring coagulation parameters should be advisable to improve the clinical management of patients with COVID-19.

\section{AUTHOR CONTRIBUTIONS}

Study concept and design: Qiang Li and Liang Chen. Data collection: Wei Xu, Ling Fei, ChenLu Huang, WeiXia Li, and XuDong Xie. Analysis and interpretation of data: Wei Xu, Ling Fei, ChenLu Huang, and Qiang Li. Drafting of the manuscript: Qiang Li. Critical revision of the manuscript: Liang Chen. 


\section{ACKNOWLEDGMENTS}

We thank all doctors who work in Shanghai Public Health Clinical Center for their efforts in the diagnosis and treatment of patients with COVID-19.

\section{CONFLICTS OF INTEREST}

The authors declare no conflicts of interest related to this study.

\section{FUNDING}

This study was supported by grant NO.19YF1441200 from Shanghai Sailing Plan Program.

\section{REFERENCES}

1. Wu F, Zhao S, Yu B, Chen YM, Wang W, Song ZG, Hu Y, Tao ZW, Tian JH, Pei YY, Yuan ML, Zhang YL, Dai FH, et al. A new coronavirus associated with human respiratory disease in China. Nature. 2020; 579:26569.

https://doi.org/10.1038/s41586-020-2008-3 PMID:32015508

2. Weekly operational update on COVID-19. Weblink: https://www.who.int/publications/m/item/weeklyepidemiological-update. Accessed November 24, 2020.

3. Gupta A, Madhavan MV, Sehgal K, Nair N, Mahajan S, Sehrawat TS, Bikdeli B, Ahluwalia N, Ausiello JC, Wan $E Y$, Freedberg DE, Kirtane AJ, Parikh SA, et al. Extrapulmonary manifestations of COVID-19. Nat Med. 2020; 26:1017-32.

https://doi.org/10.1038/s41591-020-0968-3 PMID: $\underline{32651579}$

4. Zhai Z, Li C, Chen Y, Gerotziafas G, Zhang Z, Wan J, Liu P, Elalamy I, Wang C, and Prevention Treatment of VTE Associated with COVID-19 Infection Consensus Statement Group. Prevention and Treatment of Venous Thromboembolism Associated with Coronavirus Disease 2019 Infection: A Consensus Statement before Guidelines. Thromb Haemost. 2020; 120:937-48.

https://doi.org/10.1055/s-0040-1710019 PMID:32316065

5. Adam EH, Zacharowski K, Miesbach W. A comprehensive assessment of the coagulation profile in critically ill COVID-19 patients. Thromb Res. 2020; 194:42-44.

https://doi.org/10.1016/i.thromres.2020.06.026 PMID: $\underline{32723615}$

6. Luo L, Xu M, Du M, Kou H, Liao D, Cheng Z, Mei H, Hu Y. Early coagulation tests predict risk stratification and prognosis of COVID-19. Aging (Albany NY). 2020; 12:15918-37.

https://doi.org/10.18632/aging.103581

PMID:32860672

7. Tang N, Bai H, Chen X, Gong J, Li D, Sun Z. Anticoagulant treatment is associated with decreased mortality in severe coronavirus disease 2019 patients with coagulopathy. J Thromb Haemost. 2020; 18:1094-99.

https://doi.org/10.1111/ith.14817

PMID:32220112

8. Yao Y, Cao J, Wang Q, Shi Q, Liu K, Luo Z, Chen X, Chen S, Yu K, Huang Z, Hu B. D-dimer as a biomarker for disease severity and mortality in COVID-19 patients: a case control study. J Intensive Care. 2020; 8:49.

https://doi.org/10.1186/s40560-020-00466-z PMID:32665858

9. Wiersinga WJ, Rhodes A, Cheng AC, Peacock SJ, Prescott HC. Pathophysiology, Transmission, Diagnosis, and Treatment of Coronavirus Disease 2019 (COVID-19): A Review. JAMA. 2020; 324:782-93. https://doi.org/10.1001/jama.2020.12839 PMID:32648899

10. Kaufman RM, Djulbegovic $B$, Gernsheimer $T$, Kleinman S, Tinmouth AT, Capocelli KE, Cipolle MD, Cohn CS, Fung MK, Grossman BJ, Mintz PD, O'Malley $B A$, Sesok-Pizzini DA, et al, and AABB. Platelet transfusion: a clinical practice guideline from the AABB. Ann Intern Med. 2015; 162:205-13.

https://doi.org/10.7326/M14-1589

PMID:25383671

11. Zhang $A$, Leng $Y$, Zhang $Y$, Wu K, Ji $Y$, Lei $S$, Xia Z. Meta-analysis of coagulation parameters associated with disease severity and poor prognosis of COVID19. Int J Infect Dis. 2020; 100:441-48. https://doi.org/10.1016/i.ijid.2020.09.021 PMID:32947052

12. Quintana-Díaz $M$, Andrés-Esteban EM, RamírezCervantes KL, Olivan-Blázquez B, Juárez-Vela R, GeaCaballero V. Coagulation Parameters: An Efficient Measure for Predicting the Prognosis and Clinical Management of Patients with COVID-19. J Clin Med. 2020; 9:3482.

https://doi.org/10.3390/jcm9113482

PMID:33126706

13. Shah S, Shah K, Patel SB, Patel FS, Osman M, Velagapudi P, Turagam MK, Lakkireddy D, Garg J. Elevated D-Dimer Levels Are Associated With Increased Risk of Mortality in Coronavirus Disease 2019: A Systematic Review and Meta-Analysis. Cardiol Rev. 2020; 28:295-302.

https://doi.org/10.1097/CRD.0000000000000330 PMID:33017364 
14. Wang J, Saguner AM, An J, Ning Y, Yan Y, Li G. Dysfunctional Coagulation in COVID-19: From Cell to Bedside. Adv Ther. 2020; 37:3033-39.

https://doi.org/10.1007/s12325-020-01399-7 PMID: $\underline{32504450}$

15. Delvaeye M, Conway EM. Coagulation and innate immune responses: can we view them separately? Blood. 2009; 114:2367-74. https://doi.org/10.1182/blood-2009-05-199208 PMID:19584396

16. Lipinski S, Bremer L, Lammers T, Thieme F, Schreiber $S$, Rosenstiel P. Coagulation and inflammation. Molecular insights and diagnostic implications. Hamostaseologie. 2011; 31:94-102, 104. https://doi.org/10.5482/ha-1134 PMID:21152678

17. Gustafson D, Raju S, Wu R, Ching C, Veitch S, Rathnakumar K, Boudreau E, Howe KL, Fish JE. Overcoming Barriers: The Endothelium As a Linchpin of Coronavirus Disease 2019 Pathogenesis? Arterioscler Thromb Vasc Biol. 2020; 40:1818-29. https://doi.org/10.1161/ATVBAHA.120.314558 PMID: $\underline{32510978}$

18. Helms J, Tacquard C, Severac F, Leonard-Lorant I, Ohana M, Delabranche X, Merdji H, Clere-Jehl R, Schenck M, Fagot Gandet F, Fafi-Kremer S, Castelain $\mathrm{V}$, Schneider F, et al, and CRICS TRIGGERSEP Group (Clinical Research in Intensive Care and Sepsis Trial Group for Global Evaluation and Research in Sepsis). High risk of thrombosis in patients with severe SARS-CoV-2 infection: a multicenter prospective cohort study. Intensive Care Med. 2020; 46:1089-98.

https://doi.org/10.1007/s00134-020-06062-x

PMID: $\underline{32367170}$

19. Cui S, Chen S, Li X, Liu S, Wang F. Prevalence of venous thromboembolism in patients with severe novel coronavirus pneumonia. J Thromb Haemost. 2020; 18:1421-24. https://doi.org/10.1111/ith.14830 PMID: $\underline{32271988}$
20. Llitjos JF, Leclerc $M$, Chochois C, Monsallier JM, Ramakers M, Auvray M, Merouani K. High incidence of venous thromboembolic events in anticoagulated severe COVID-19 patients. J Thromb Haemost. 2020; 18:1743-46.

https://doi.org/10.1111/jth.14869

PMID: 32320517

21. Lodigiani C, lapichino $G$, Carenzo L, Cecconi $M$, Ferrazzi P, Sebastian T, Kucher N, Studt JD, Sacco C, Bertuzzi A, Sandri MT, Barco S, and Humanitas COVID19 Task Force. Venous and arterial thromboembolic complications in COVID-19 patients admitted to an academic hospital in Milan, Italy. Thromb Res. 2020; 191:9-14.

https://doi.org/10.1016/j.thromres.2020.04.024 PMID:32353746

22. Paranjpe I, Fuster V, Lala A, Russak AJ, Glicksberg BS, Levin MA, Charney AW, Narula J, Fayad ZA, Bagiella E, Zhao S, Nadkarni GN. Association of Treatment Dose Anticoagulation With In-Hospital Survival Among Hospitalized Patients With COVID-19. J Am Coll Cardiol. 2020; 76:122-24.

https://doi.org/10.1016/i.jacc.2020.05.001 PMID: 32387623

23. Tafur AJ, McBane R 2nd, Wysokinski WE, Litin S, Daniels P, Slusser J, Hodge D, Beckman MG, Heit JA. Predictors of major bleeding in peri-procedural anticoagulation management. J Thromb Haemost. 2012; 10:261-67.

https://doi.org/10.1111/j.1538-7836.2011.04572.x PMID:22123000

24. Li Q, Xu W, Li WX, Huang CL, Chen L. Dynamics of cytokines and lymphocyte subsets associated with the poor prognosis of severe COVID-19. Eur Rev Med Pharmacol Sci. 2020; 24:12536-44.

https://doi.org/10.26355/eurrev 20201224051 PMID:33336774 